\title{
On the Problem of Unbalanced Load Distribution in Wireless Sensor Networks
}

\author{
Mark Perillo, Zhao Cheng, and Wendi Heinzelman \\ Department of Electrical and Computer Engineering \\ University of Rochester \\ Rochester, NY 14627 \\ Email: $\{$ perillo, zhcheng, wheinzel $\}$ ece.rochester.edu
}

\begin{abstract}
In multi-hop wireless sensor networks that are characterized by many-to-one traffic patterns, problems related to energy imbalance among sensors often appear. When each node has a fixed transmission range, the amount of traffic that sensor nodes are required to forward increases dramatically as the distance to the data sink becomes smaller. Thus, sensors closest to the data sink tend to die early, leaving areas of the network completely unmonitored and causing network partitions. Alternatively, if all sensors transmit directly to the data sink, the furthest nodes from the data sink will die much more quickly than those close to the $s$ ink. While it may seem that network lifetime could be improved by use of a more intelligent transmission power control policy that balances the energy used in each node by requiring nodes further from the data sink to transmit over longer distances (although not directly to the data sink), such a policy can only have a limited effect. In fact, this energy balancing can be achieved only at the expense of gross energy inefficiencies. In this paper, we investigate the transmission range distribution optimization problem and show where these inefficiencies exist when trying to maximize the lifetime of many-to-one wireless sensor networks.
\end{abstract}

\section{INTRODUCTION}

Large scale wireless sensor networks have recently gained attention for their multitude of potential applications. Since microsensors typically operate on batteries and are thus limited in their active lifetime, the problem of designing protocols to achieve energy efficiency to extend network lifetime has become a major concern for network designers. It is also important to maintain a balance of power consumption in the network so that certain nodes do not die much earlier than others, leading to unmonitored areas in the network.

Previous research has shown that because of the characteristics of wireless channels, multi-hop forwarding between a data source and a data sink is often more energy efficient than direct transmission. Based on the power model of a specific sensor node platform, there exists an optimal transmission range that minimizes overall power consumption in the network. When using such a fixed transmission range in many-to-one sensor network applications, however, an energy imbalance problem manifests itself, as a "hot spot" is created around the data sink, or base station. The nodes in this "hot spot" are required to forward an unproportionately high amount of traffic and typically die at a very early stage.

Intuition leads us to believe that the "hot spot" problem can be solved by varying the transmission range among nodes at different distances to the base station so that energy consumption can be more evenly distributed and the lifetime of the network can be extended. However, this is only true to some extent, as energy balancing can only be achieved at the expense of using the energy resources of some nodes inefficiently. In this paper, we formulate the transmission range distribution optimization problem, and present a general optimization model to analyze the limits of network lifetime for uniformly deployed, many-to-one wireless sensor networks. In contrast to transmission power optimization problems where a fixed network-wide transmission range is assigned to every node, we allow sensors to set their individual transmission ranges according to their locations. Furthermore, their traffic may be distributed over multiple distances.

The rest of this paper is organized as follows. Section II addresses related work. Section III models the transmission range distribution optimization problem. Section IV provides some insight into the problem through simulation results. Section $\mathrm{V}$ concludes the paper and suggests future work in this area.

\section{RELATED WORK}

Early work in transmission range optimization assumed that forwarding data packets towards a data sink over many short hops is more energy efficient than forwarding over a few long hops, due to the nature of wireless communication. The problem of setting transmission power to a minimal level that will allow a network to remain connected has been considered in several studies [1], [2]. Later, others noted that because of the electronics overhead involved in transmitting packets, there exists an optimal non-zero transmission range, at which power efficiency is maximized [3], [4]. The goal of these studies is to find a fixed network-wide transmission range. However, using such schemes may result in extremely unbalanced energy consumption among the nodes in sensor networks characterized by many-to-one traffic patterns. The goal of our work is to investigate whether or not individual nodes can vary their transmission range as a function of their distance to the data sink and optimally distribute their traffic so that network lifetime is maximized. The work of [5] is the most similar to ours that we know of, in that the authors attempt to optimize each sensor's transmission range in a many-to-one wireless sensor network. However, we model the 
problem differently in several aspects, most notably in that we assume constant node density throughout the network and the ability to distribute traffic over multiple distances. Thus, we are solving a different problem in our work and the results presented here and in [5] are not directly comparable.

\section{TRANSMisSion RANGE Distribution Optimization PROBLEM}

In this section, we formalize the transmission range distribution optimization problem, which is solved by determining how a node should distribute its outgoing data packets over multiple distances, always using the minimum transmission power necessary to send over each distance. Given the energy constraints and data generation rate of each sensor node, the lifetime of the network, which we define to be the time at which the first sensor death occurs, can be maximized by using this optimal distribution. In typical sensor network applications, it may be true that the network can survive node failures as long as neighboring sensors can assume the failing nodes' responsibilities; however, we expect neighboring nodes to exhibit similar trends and thus attain similar lifetimes. Thus, we consider our definition of network lifetime valid even for such sensor network models. We refer to the problem addressed in this work as a transmission range distribution optimization problem rather than a transmission range optimization problem because we assume that nodes may send packets over multiple transmission ranges instead of setting a fixed transmission range.

\section{A. Assumptions}

In our model, we make several basic assumptions. First, we assume that the power consumption of sensor nodes is dominated by communication costs, as opposed to sensing and processing costs. This assumption is reasonable for many types of sensor nodes that require very little energy, such as pressure and temperature sensors. We also ignore the overhead that would typically be introduced by the routing layer. However, for long lasting sensor networks with little or no mobility, route updates should be performed infrequently and should not significantly affect the overall power consumption in the network.

We have also ignored any potential overhead at the MAC layer. Due to the scarce energy supplies in sensor nodes, many groups have proposed the use of coordinated TDMA scheduling in the MAC layer. Because of the low data rates expected in many sensor network applications, even localized TDMA scheduling (as opposed to globally coordinated scheduling) should not cause much communication overhead in the form of collisions and necessary retransmissions. Furthermore, TDMA scheduling can eliminate most overhead introduced by idle listening and overhearing. As with the overhead associated with routing updates, the establishment of schedules can take place very infrequently and should not contribute significantly to overall power consumption.

Another assumption that we make is that a node's transmission range is unlimited. While this assumption may seem to be impractical, it should be noted that the focus of our work is to analyze the fundamental limitations of sensor network lifetime. In the case of optimistic results stemming from this analysis (i.e., that the "hot spot" problem can be effectively solved through transmission range distribution optimization), this assumption will need to be relaxed so that more practical policies can be found. In the case of more pessimistic results (i.e., that the "hot spot" problem cannot be solved), this assumption will strengthen the results.

Our final assumption is that the channels are lossless. We can assume that the minimum energy necessary to transmit over a link is the minimum energy such that the packet loss rate is below some small threshold. However, it may be possible to reduce transmission energy further and introduce some nonzero packet loss rate as long as overall power savings are achieved. These factors may contribute to interesting effects, and we intend to observe these effects in future work.

\section{B. Problem Modeling}

In our network model, a set of $N$ sensors is deployed in a region in order to monitor some physical phenomenon. We refer to the complete set of sensors that has been deployed as $S=\left\{S_{1} \ldots S_{N}\right\}$. The power consumption model that we use is such that the amount of energy to transmit a bit can be represented as $E_{b i t, t x}=E_{\text {elec }}+\epsilon \times d^{2}$, and the amount of energy to receive a bit can be represented as $E_{b i t, r x}=E_{\text {elec }}$, where $E_{\text {elec }}$ represents the electronics energy and the transmitter amplifier's energy consumption is characterized by $\epsilon$ [6]. Nodes generate traffic at a rate of $R_{g}\left(S_{i}\right)$ bits per second. All data must eventually reach a single data sink, or base station, labeled $S^{*}$.

The goal of our problem is to find a transmission range distribution that determines what fraction of packets each node should send over each distance. This is equivalent to creating a probability density function of the transmission range for each sensor node. If the total traffic being forwarded from sensor $S_{i}$ to sensor $S_{j}$ is denoted as $T\left(S_{i}, S_{j}\right)$ and the distance between $S_{i}$ and $S_{j}$ is denoted as $d\left(S_{i}, S_{j}\right)$, the transmission range distribution $p_{i}(d)$ of sensor $S_{i}$ can be represented as

$$
p_{i}(d)=\frac{\sum_{S_{j}: d\left(S_{i}, S_{j}\right)=d} T\left(S_{i}, S_{j}\right)}{\sum_{S_{j} \in S} T\left(S_{i}, S_{j}\right)}
$$

The first constraint of our problem, related to the conservation of data flow at all sensor nodes, is

$$
\begin{aligned}
& \sum_{S_{j} \in S} T\left(S_{j}, S_{i}\right)+R_{g}\left(S_{i}\right)= \\
& \sum_{S_{j} \in S} T\left(S_{i}, S_{j}\right)+T\left(S_{i}, S^{*}\right)
\end{aligned} \quad \forall S_{i} \in S
$$

Applying the incoming and outgoing traffic to our power model, the power consumption at node $S_{i}$ can be expressed as

$$
\begin{aligned}
& P\left(S_{i}\right)=E_{\text {elec }} \sum_{S_{j} \in S} T\left(S_{j}, S_{i}\right)+ \\
& \left(\sum_{S_{j} \in S} T\left(S_{i}, S_{j}\right)+T\left(S_{i}, S^{*}\right)\right) \times \\
& \left(E_{\text {elec }}+\epsilon \sum_{S_{j} \in S} p_{i}\left(d\left(S_{i}, S_{j}\right)\right) d^{2}\left(S_{i}, S_{j}\right)\right)
\end{aligned}
$$




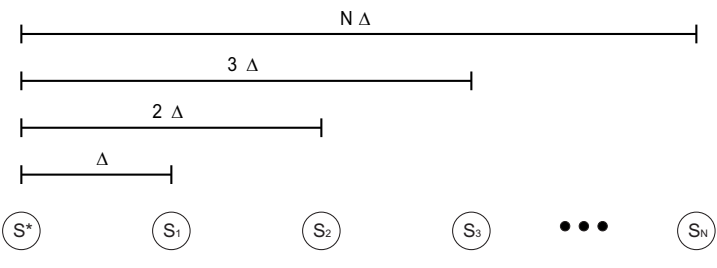

Fig. 1. A chain topology network modeled in a one-dimensional space. Nodes are equally spaced in this scenario.

Given each sensor $S_{i}$ 's initial energy $E_{\text {initial }}\left(S_{i}\right)$, the network lifetime $L$ must satisfy

$$
L \times P\left(S_{i}\right) \leq E_{\text {initial }}\left(S_{i}\right) \quad \forall S_{i} \in S
$$

The problem that we are addressing in this paper is to find a distribution of the (multi-hop) data transmissions that maximizes $L$, defined as the time when the first node's energy supply has been depleted. In other words, we find values of $p_{i}(d)$ that maximize $L$ subject to the constraints of Equations 2 and 4.

Note that this is effectively solving the problem

$$
\max \min \frac{E_{\text {initial }}\left(S_{i}\right)}{P\left(S_{i}\right)}
$$

We can solve the problem through a linear programming approach using the constraints of Equations 2 and 4, and the goal of maximizing sensor network lifetime $L$. The linear program finds the optimal values of $T\left(S_{i}, S_{j}\right)$, which we can map to $p_{i}(d)$, for given values of $E_{\text {elec }}$ and $\epsilon$ and a given network topology. $\mathrm{S}$

\section{Simulations}

In this section, we provide simulation results in order to observe the optimal transmission range distributions for several typical sensor network deployment scenarios. In all simulations, we used values of $E_{\text {elec }}=50 \mathrm{~nJ} / \mathrm{bit}$ and $\epsilon=10 \mathrm{pJ} / \mathrm{bit} / \mathrm{m}^{2}$ in the power model. According to this power model and the analysis provided in [3], [4], the ideal transmission range for nodes in a general ad hoc network would be $100 \mathrm{~m}$. In other words, $100 \mathrm{~m}$ is the most power efficient operating point in the absence of the sensor networkspecific "hot spot" problem. The initial energy and data generation rate of all nodes in the network were arbitrarily set to $1 J$ and $1 \mathrm{bit} /$ second, respectively, in all simulations.

\section{A. Chain Scenario}

First, we observe a special case of the transmission range distribution optimization problem where the topology consists of a chain of sensors, separated by a distance of $\Delta$, leading to the base station. This scenario may occur in such applications as highway traffic congestion monitoring. The scenario is depicted in Figure 1.

The optimal transmission range distribution for a chain network with node spacing of $5 \mathrm{~m}$ and a radius of $500 \mathrm{~m}$ is shown in Figure 2. Figure 2(a) shows a three-dimensional

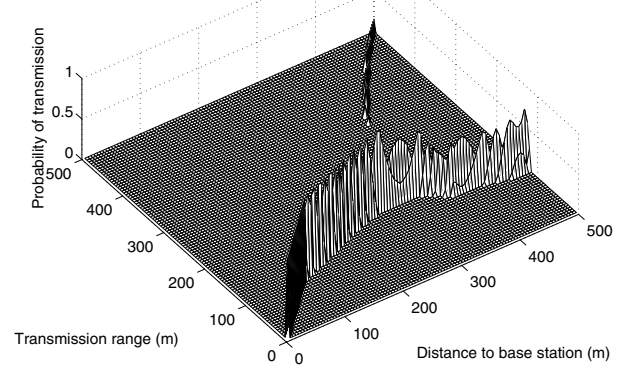

(a)

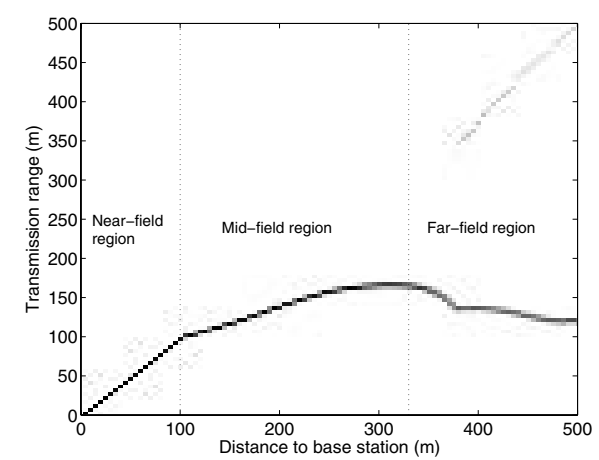

(b)

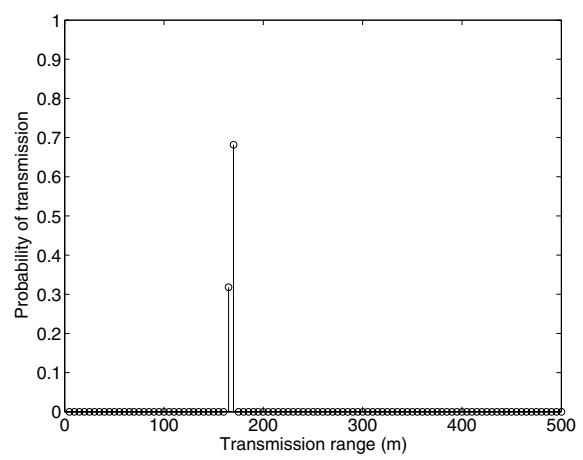

(c)

Fig. 2. Optimal transmission range distribution for a chain topology network with a $500 \mathrm{~m}$ radius and $5 \mathrm{~m}$ spacing between nodes. Figure (b) is simply the view of Figure (a) from above. Figure (c) shows the transmission range probability density function of the node located $300 \mathrm{~m}$ from the base station.

plot of the transmission range distribution, while Figure 2(b) illustrates the same information in an image, which will be the format used to represent our results for the remainder of this paper. Dark areas in the distribution images refer to areas of dense transmission probability. By taking a vertical cross section of the image data, as shown in Figure 2(c) for a node at a distance of $300 \mathrm{~m}$ from the base station, we can more easily interpret the data. A cross section gives us the probability density function $p_{i}\left(S_{i}\right)$ for the sensor $S_{i}$ located at the position where the cross section is taken.

As shown in Figure 2(b), the sensors can be separated into three regions: a near-field, a mid-field, and a far-field. In the near-field region, nodes transmit directly to the base station. 
Nodes at farther distances, in the mid-field region, transmit most of their packets over multiple hops, and the distribution of the packets is concentrated at a distance that increases approximately linearly with distance from the base station. Packets from nodes in the far-field region seem to be split between being sent over an energy efficient transmission range and directly to the base station.

While extending lifetime is the primary goal of the transmission range distribution optimization problem, energy usage should not be ignored. Lifetimes approaching that of the optimal solution can often be attained when using only a fraction of the total network-wide energy available. If we allocate the same amount of initial energy to each individual node but use just a fraction of the total network energy, we find an interesting trend in the attainable lifetime, shown in Figure 3. In fact, as the total energy consumed in the network decreases from $100 \%$, changes in the lifetime are initially very limited. Figure 4 illustrates the changes in the optimal transmission range distribution and power consumption for each node at different points on the energy-lifetime curve. The energy inefficiency is caused primarily by the nodes in the farfield region and the nodes at the border of the near-field and the mid-field regions.

The inefficiency of the transmissions in the far-field region can be explained as follows. Nodes closer to the base station in general must forward more traffic than those far away. The nodes in the farthest region need to send only their own traffic, and so in an energy efficient solution (in which they transmit over reasonably small transmission ranges), they consume the least energy. In order to balance the energy and increase lifetime by a minimal amount, they may take several of the packets that were sent over the "reasonable" transmission ranges and send them over longer distances (e.g., directly to the base station), thus reducing the load on the intermediate nodes. However, because of the long distances, the number of additional packets that are sent over longer distances rather than using the "reasonable" transmission ranges is limited. As the plots in Figure 4 show, packets that are sent over longer distances rather than using the "reasonable" transmission ranges are the primary ones to be transferred to a more energy efficient range (see the regions of the distributions enclosed by the dashed lines as well as the energy consumption plots) as the total network-wide energy consumption drops from $100 \%$ to $90 \%$.

Nodes at the border of the near-field and mid-field regions also contribute to the energy inefficiency by transmitting packets to nodes located at very small distances from the base station. Consider a node far from the base station that sends its packets to a node located close to the base station. The total energy consumption for such packet transmissions would be slightly higher than the energy consumption to send packets directly to the base station. However, in the absence of these two-hop packet transmissions, the farther node consumes more energy than the closer node and is more of a limiting factor in terms of network lifetime. Thus, while requiring the nearer node to forward traffic increases total energy consumption,

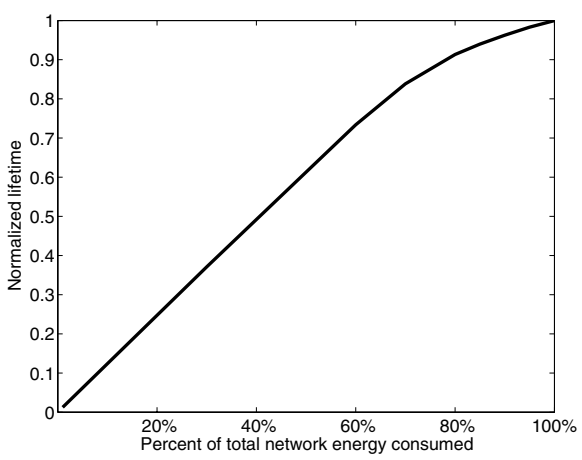

Fig. 3. Lifetime vs. percentage of total network energy consumed.
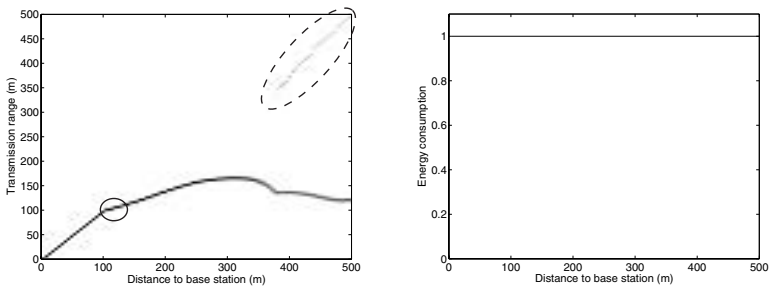

(a)
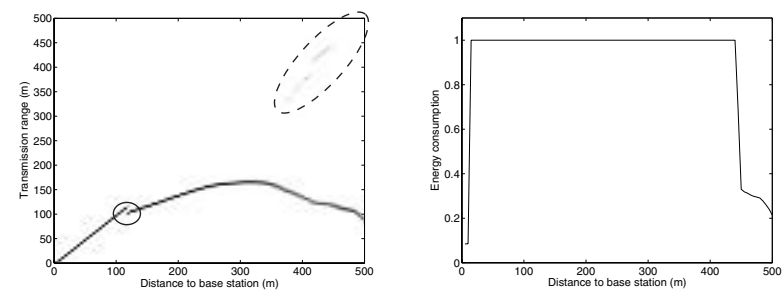

(b)

Fig. 4. Optimal transmission range distribution and energy consumption as a function of distance for $100 \%$ (a) and $90 \%$ (b) of total network energy consumed.

it minimizes the maximum energy consumption among the nodes and is included in the optimal solution. Figure 4 (see the regions enclosed by the solid lines) illustrates that as the total network-wide energy consumption drops from $100 \%$ to $90 \%$, some of the packets that would have been sent over a long-hop followed by a short-hop are instead sent over a single slightly longer hop. Note that the reduction in energy consumption actually occurs at the nodes that would have received these packets (i.e., those closest to the base station), rather than at the nodes that would have sent them.

Next, we study the impact of node density on the optimal transmission distribution, which we expected would be minimal. As density increases, the ability of individual nodes to rotate activity or generate data at a lesser rate lengthens lifetime if intelligent schemes are used; however, if the density is increased uniformly throughout the network, the additionally deployed nodes should exhibit similar trends in terms of transmission range distribution. Figure 5, which shows the optimal transmission range distribution for networks with a radius of $500 m$, with $5 m, 10 m, 20 m$, and $100 m$ spacing 


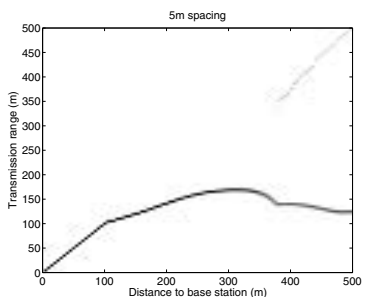

(a)

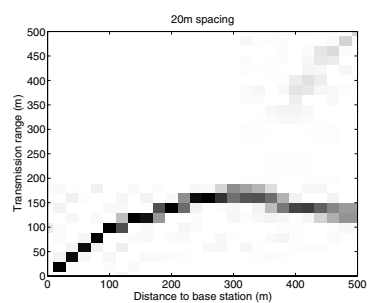

(c)

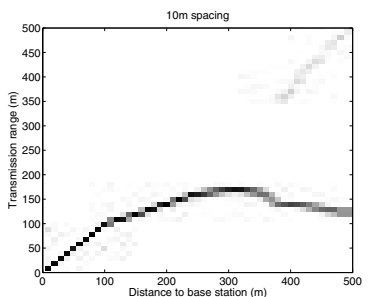

(b)

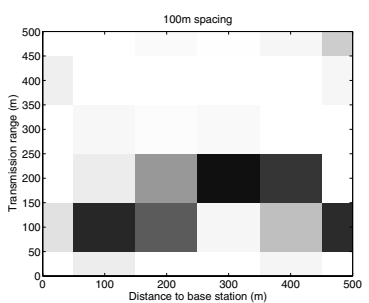

(d)
Fig. 5. Optimal transmission range distribution as a function of distance from the base station for $5 m$ (a), $10 m$ (b), $20 m$ (c), and $100 m$ (d) spacing, $500 \mathrm{~m}$ radius.

between the nodes, verifies our intuition. The only anomaly is in the $100 \mathrm{~m}$ spacing scenario, where the large spacing prevents transmissions over optimal distances from being realized. In scenarios with higher node density, the optimal transmission range distributions are very similar, as expected.

Next, we observe the effect of network radius on network lifetime and optimal transmission range distribution. In Figure 6 , we show the optimal transmission range distribution as we set the network radius at $250 \mathrm{~m}$ and $1000 \mathrm{~m}$, using $5 \mathrm{~m}$ sensor spacing. As the figure shows, increasing the network radius mostly extends the far-field region, while the size of the nearfield and mid-field regions vary only slightly. The energylifetime curve is plotted in Figure 7. In larger networks, the farthest nodes must operate at much more inefficient points in order to balance energy consumption and so inefficiency occurs earlier as the percentage of network energy consumed increases.

\section{B. Two-Dimensional Sensor Fields}

Next, we consider the scenario of a two-dimensional sensor field, with a base station located in the center of the field. We modeled a dense two-dimensional field as a one-dimensional field with nonuniform spacing. With infinitely dense sensor deployment, we can assume that sensors will always send their packets within an infinitesimally thin angle toward the base station, as shown in Figure 8(a). Since the number of nodes $N$ within the network radius $R$ satisfies $N \propto R^{2}$ for twodimensional networks, when mapped into the one-dimensional space, the distance of a node to the base station should be proportional to the square root of the node index, as shown in Figure 8(b).

We ran simulations to find the optimal transmission range distribution for a two-dimensional sensor field. In these simulations, the number of nodes in the network was kept constant

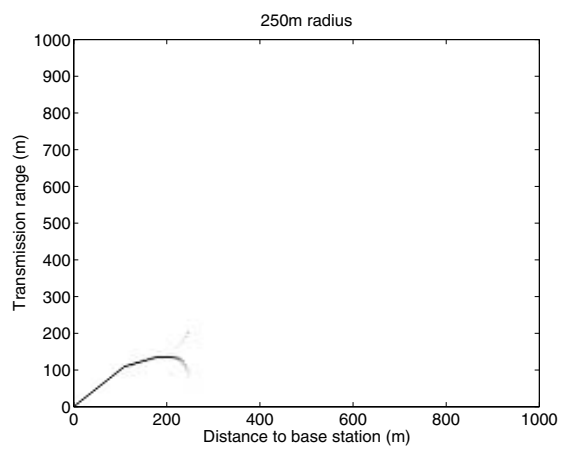

(a)

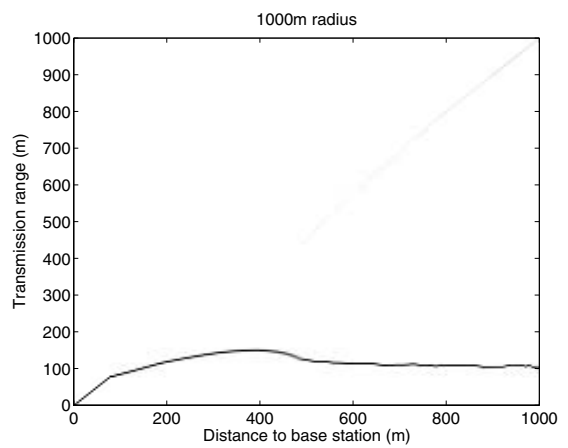

(b)

Fig. 6. Optimal transmission range distribution as a function of distance from the base station for radius of $250 \mathrm{~m}$ (a) and $1000 \mathrm{~m}$ (b), $5 \mathrm{~m}$ sensor spacing.

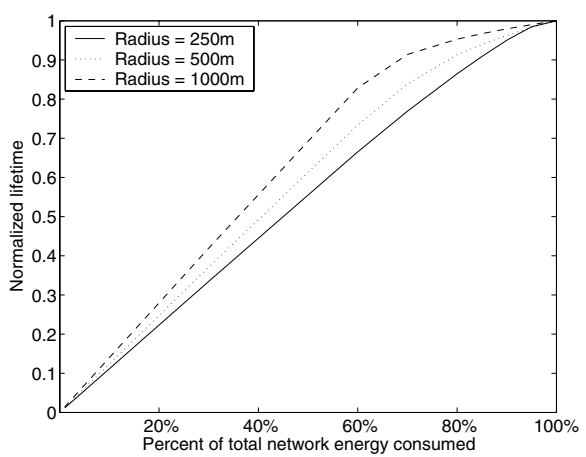

Fig. 7. Lifetime vs. energy consumption tradeoff for network radius of $250 \mathrm{~m}, 500 \mathrm{~m}$, and $1000 \mathrm{~m}$.

and their spacing scaled up according to the network radius. The optimal transmission range distributions are shown in Figure 9 for network radii of $250 \mathrm{~m}$ and $1000 \mathrm{~m}$. The trends seem similar to those in the chain scenario, although it seems that a larger portion of the packets are sent over long distances. This is to be expected since there are proportionally fewer nodes near the base station to route proportionally more data from nodes farther from the base station. Since it is inefficient to send packets over long distances, we can expect the energylifetime curve to reflect higher inefficiency at points of high energy consumption. Figure 10 verifies this and shows that 


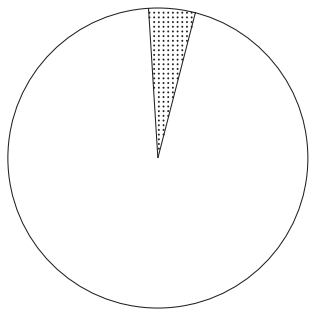

(a)

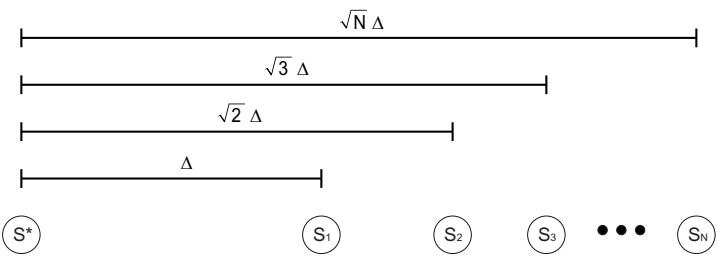

(b)

Fig. 8. Two-dimensional sensor field (a) and one-dimensional modeling (b).

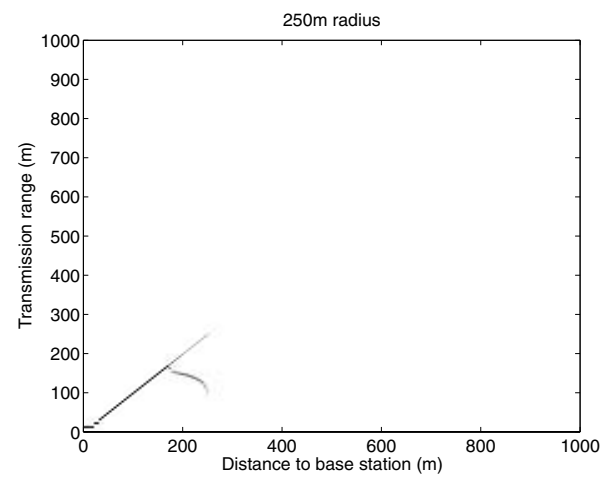

(a)

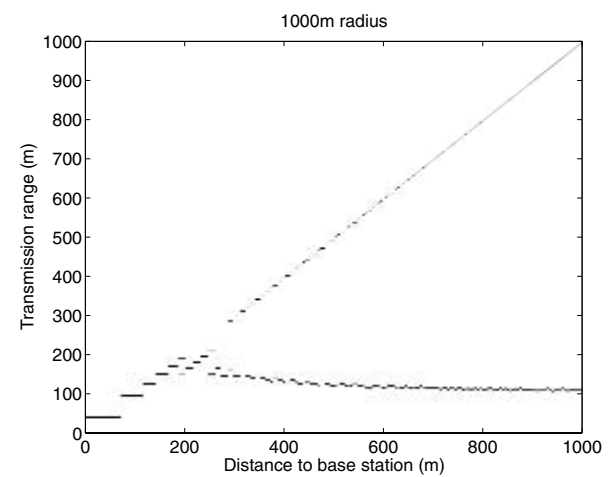

(b)

Fig. 9. Optimal transmission range distribution as a function of distance from the base station for a two-dimensional sensor field with radius of $250 \mathrm{~m}$ (a) and $1000 m$ (b).

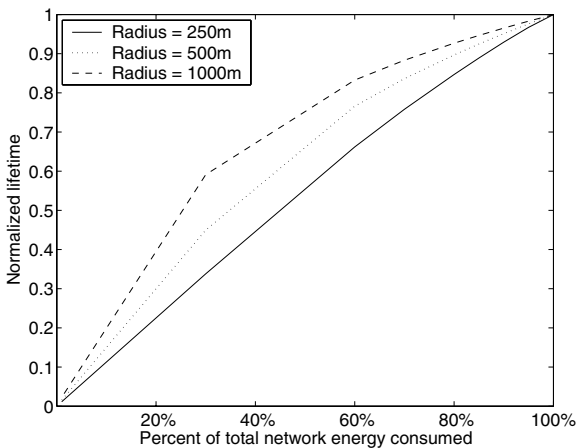

Fig. 10. Lifetime vs. percentage of the total energy consumed in the network for a two-dimensional sensor field with various network radii.

inefficiency occurs at even lower points of total network-wide energy consumption than in the chain scenario, especially for large networks. We have observed similar results in randomly deployed two-dimensional sensor networks. However, we have omitted these results due to space limitations.

\section{CONCLUSIONS}

We have proposed a general model to study the optimal transmission range distribution that allows the lifetime of sensor networks to be maximized. Based on this model, we revealed the upper bound of the lifetime of several typical scenarios and demonstrated the inability to make good use of the energy of nodes furthest from the base station, even when utilizing the optimal distribution. Thus, even under the most ideal scenario (e.g., unlimited transmission ranges), varying the tranmission power of individual nodes cannot alone solve the "hot spot" problem. One way to circumvent the "hot spot" problem is through rotation of the data sink, an approach that is used by such protocols as LEACH [6]. One potential area of future work is to modify the energy constraints so as to limit only the total energy consumed in the network. Solutions to this modified problem could have implications related to sensor deployment.

\section{REFERENCES}

[1] V. Rodoplu and T. Meng, "Minimum energy mobile wireless networks," in Proceedings of the IEEE International Conference on Communications, 1998.

[2] R. Ramanathan and R. Hain, "Topology control of multihop wireless networks using transmit power adjustment," in Proceedings of the Nineteenth International Annual Joint Conference of the IEEE Computer and Communications Societies (INFOCOM), 2000.

[3] Y. Chen, E. Sirer, and S. Wicker, "On selection of optimal transmission power for ad hoc networks," in Proceedings of the Thirty-Sixth Hawaii International Conference on System Sciences (HICSS-36), 2003.

[4] J. Deng, Y. Han, P. Chen, and P. Varshney, "Optimum transmission range for wireless ad hoc networks," in Proceedings of the IEEE Wireless Communications and Networking Conference (WCNC), 2004.

[5] I. Howitt and J. Wang, "Energy balanced chain in wireless sensor networks," in Proceedings of the IEEE Wireless Communications and Networking Conference (WCNC), 2004.

[6] W. Heinzelman, A. Chandrakasan, and H. Balakrishnan, "An applicationspecific protocol architecture for wireless microsensor networks," IEEE Transactions on Wireless Communications, vol. 1, no. 4, pp. 660-670, Oct. 2002. 